\title{
Radiosensitization Effect of Overexpression of Adenovirus- mediated SIRT6 on A549 Non-small Cell Lung Cancer Cells
}

\author{
Yong Cai, Zhao-Ying Sheng, Shi-Xiong Liang*
}

\begin{abstract}
Objective: To explore the radiosensitization effect of overexpression of silent information regulator 6 (SIRT6) on A549 non-small cell lung cancer (NSCLC) cells. Methods: Adenovirus vector Ad-SIRT6 causing overexpression of SIRT6 was established. Western blotting and MTT assay were adopted to detect the level of SIRT6 protein and the inhibitory rate of A549 cell proliferation after different concentrations of adenovirus transduction ( 0 , 25, 100, 200, and $400 \mathrm{pfu} / \mathrm{cell}$ ) for $24 \mathrm{~h}$. Control group, Ad-null group and Ad-SIRT6 group were designed in this experiment and virus concentration of the latter two groups was $200 \mathrm{pfu} / \mathrm{cell}$. Colony formation assays were employed to test survival fraction (SF) of the 3 groups after $0,2,4,6,8,10 \mathrm{X}$-ray irradiation. Flow cytometry was used to detect the status of cell cycle of 3 groups after $48 \mathrm{~h}$ of $4 \mathrm{~Gy}$ X-ray irradiation and Western blotting was used to determine the expression of apoptosis-related genes of 3 groups after $48 \mathrm{~h}$ of $4 \mathrm{GyX}$-ray irradiation. Results: In the range of 25 400 pfu/cell, the inhibitory rate of A549 cell proliferation increased as adenovirus concentration raised. The inhibitory rates under the concentrations of $0,25,100,200$, and $400 \mathrm{pfu} / \mathrm{cell}$ were $0 \%$, $4.23 \pm 0.34 \%, 12.7 \pm 2.57 \%, 22.6 \pm 3.38 \%, 32.2 \pm 3.22 \%, 38.7 \pm 4.09 \%$ and $47.8 \pm 5.58 \%$ and there were significantly differences among groups $(P<\mathbf{0 . 0 5})$. SF in Ad-SIRT6 group was lower than Ad-null and control groups after 4 10Gy $X$-ray irradiation $(P<0.05)$ and the sensitization enhancement ratio (SER) was 1.35 when compared with control group. Moreover, after $48 \mathrm{~h}$ of $4 \mathrm{~Gy} \mathrm{X}$-ray irradiation, there appeared a significant increase in G1-phase cell proportion, up-regulated expression of the level of apoptosis-promoting genes (Bax and Cleaved caspase-3), but a obvious decline in S-phase and G2-phase cell proportion and a significant decrease of the level of apoptosisinhibiting gene (Bal-2) in the Ad-SIRT6 group $(P<0.05)$. Conclusion: The over-expression of adenovirus-mediated SIRT6, which has radiosensitization effect on A549 cells of NSCLC, can inhibit the proliferation of A549 cells and cause G0/G1 phase retardation as well as induce apoptosis of cells.
\end{abstract}

Keywords: Silent information regulator 6 - overexpression - non-small cell lung cancer - radiosensitization effect

Asian Pac J Cancer Prev, 15 (17), 7297-7301

\section{Introduction}

Non-small cell lung cancer (NSCLC), a common type of lung cancer with poor prognosis, is one of the main causes of cancer death all over the world (Jemal et al., 2010). Patients with locally advanced NSCLC who can't receive surgery account for approximately $40 \%$ (Vokes, 2007), thus, for whom, although radiotherapy is a commonly-used treatment, adverse reactions caused by high-dose radiotherapy and radioresistance of tumor tissue limit the curative effects, so the valid radiosensitivityenhancing measures need to be explored. The change of sugar metabolic pathway is the common behavior of tumor cells which lays the foundation of macromolecular synthesis for the growth and proliferation of tumor cells (Vander et al., 2009). Multiple studies have revealed that down-regulated expression of lactic dehydrogenase (LDH) and pyruvate dehydrogenase kinase-1 (PDHK-1) can inhibit the growth and proliferation of the tumor (Bonnet et al., 2007; Fantin, et al., 2006; Le et al., 2010), indicating that the change of tumor cell metabolism is of great significance to tumor cell growth and proliferation, which now has become a hot issue of prevention and treatment of the tumor. SIRT6 is NAD+-dependent histone acetylation enzyme regulating multiple physiological processes of cells such as the growth and apoptosis of cells (Zhong et al., 2010). A study in Cell in 2012 elaborated cells with the defect of SIRT6 had oncogenicity, and SIRT6 could regulate the tumor metabolism and be considered as tumor suppressor gene (Sebastián et al., 2012). Therefore, in this study, the radiosensitization effect of overexpression of SIRT6 on A549 cells of NSCLC are explored and the results are reported as follows.

\section{Materials and Methods}

Reagent

A549 cell line was purchased from American Type Culture Collection (ATCC). Methyl thiazolyl tetrazolium (MTT), dimethylsulfoxide (DMSO), methylene double 
acryloyl, lauryl sodium sulfate and Tris were available from Sigma-Aldrich. Glycine, RNase and acrylamide were provided by Amresco Inc. Company. Cell lysis buffer, Protein Quantitative Reagent Kit-BCA Method and ECL Chemiluminescence Detection Kit were bought from Beyotime Institute of Biotechnology. RPMI 1640 medium and trypsin were purchased from Gibco and penicillin, streptomycin and fetal calf serum (FCS) from Hyclone Company, PI Cell Cycle Detection Kit from Nanjing KeyGEN Biotech. Co., Ltd, Rabbit anti-GAPDH, Rabbit anti-Bal-2 and Sheep anti-Cleaved Caspase-3 Polyclonal Antibody from Cell Sigaling Technology, Inc., Rabbit anti-Bax and Sheep anti-SIRT6 Polyclonal Antibody from Santa Cruz Biotechnology, Inc., adenovirus vector Ad-SIRT6 of overexpressed SIRT6 from SinoGenoMax Co., Ltd.

\section{Transfection efficiency test}

Western bloting was used to detect the expression of SIRT6 after $24 \mathrm{~h}$ of Ad-SIRT6 transfection. Logarithmic phase A549 cells after digested by $0.25 \%$ trypsin were innoculated in 6 -well culture plate, $1 \times 10^{6}$ in each well, collected after via the different levels of adenovirus transduction $(0,25,100,200$, and $400 \mathrm{pfu} / \mathrm{cell})$ for $24 \mathrm{~h}$, added with cell lysis buffer for cell lysis on the ice. The concentration of extractive protein was detected by BCA method and the equivalent protein supernatant taken from each well was performed with $10 \%$ SDS-polyacrylamide gel electrophoresis (SDS-PAGE), added with the proper quantity of first antibody of SIRT6 with dilution rate being 1: 200 for overnight incubation at $4^{\circ} \mathrm{C}$, then added with the sencond antibody of SIRT6 with dilution rate being 1: 3000 for $2 \mathrm{~h}$ of incubation at, and lastly procedded by ECL developing. Image-Pro Plus 5.1 software was used to analyze optical density of developing bands and the last result was expressed as the ratio of SIRT6 to internal reference GAPDH band optical density.

\section{Detection of cell proliferation}

Logarithmic phase A549 cells were prepared to singlecell suspension, which was incubated into 96-well culture plate, $1 \times 10^{5}$ each well, $200 \mu \mathrm{L}$ and after cultivation for 24 $\mathrm{h}$, transduced by the different levels of adenovirus $(0,25$, 100,200 , and $400 \mathrm{pfu} / \mathrm{cell}$ ), then after cultured with $5 \%$ $\mathrm{CO}_{2}$ at $37^{\circ} \mathrm{C}$ for $24 \mathrm{~h}$, added with $10 \mu \mathrm{MTT}(5 \mathrm{mg} \bullet \mathrm{mL}-1)$ for cultivation and stopped after $4 \mathrm{~h}$. Absorbance A value in groups was detected by ELIASA at $492 \mathrm{~nm}$ wavelength. The concentration of $0 \mathrm{pfu} / \mathrm{cell}$ was used as control group while the other concentrations were considered as experiemental group. Each concentration was designed with 6 parallel repeated wells and the experiment was repeated for 3 times. According to fomula for caculating the inhibitory rate of cell proliferation, inhibitory rate $(\%)$ $=(\mathrm{A}$ value of control group $-\mathrm{A}$ value of experimental group)/A value of control group $\times 100 \%$.

\section{Conditions of cell irradiation}

Electron linear accelerator was employed to irradiate cells. Below the cell culture plate was $5 \mathrm{~cm}$ thick water tank and above the cell culture plate was $1.55 \mathrm{~cm}$ equivalent tissue gel. The source-target distance was $100 \mathrm{~cm}$ and the field of $10 \mathrm{~cm} \times 10 \mathrm{~cm}$ was given $6 \mathrm{MV}$-X irradiation with dosage rate of $200 \mathrm{cGy} / \mathrm{min}$. Cells after given different dose of irradiation were put into the incubator with $5 \%$ $\mathrm{CO}_{2}$ and saturation humidity for cultivation at $37^{\circ} \mathrm{C}$.

\section{Clone formation experiment}

A549 single-cell suspension was prepared to be inoculated into 24 -well plate according to the cell density of 200/well and divided into control group, Ad-null group and Ad-SIRT6 group. The latter two groups with virus concentration of $200 \mathrm{pfu} /$ cell were irradiated by $0,2,4$, 6,8 and $10 \mathrm{~Gy} \mathrm{X}$-ray after $24 \mathrm{~h}$ of cell transfection, 10 d later, fixed by absolute ethyl alcohol for $15 \mathrm{~min}$ and then performed with crystal violet staining for $20 \mathrm{~min}$. The number of cell cloning more than 50 was counted under the inverted microscope for calculating the cloning efficiency $(\mathrm{CE})$ : $\mathrm{CE}(\%)=($ Clone formation average of treatment group/Inoculated cell number $) \times 100 \%$. Survival fraction $(\mathrm{SF})=($ irritated group $\mathrm{CE} /$ non-irradiated group $\mathrm{PE}) \times 100 \%$. The experiment was repeated 3 times for calculating the average. According to multi-target singlehit model [SF=1- (1-e-D/D0)N], cell survival curve was drawn for calculating the sensitization enhancement ratio (SER). SER = control group D0/Ad-SIRT6 group D0.

\section{Detection of cell cycle}

A549 cells were inoculated into 6-well plate with the density of $1 \times 10^{6} /$ well and the grouping was the same as the above-mentioned clone formation experiment. After transduced by the corresponding viral vector and irradiated by $4 \mathrm{~Gy} \mathrm{X}$-ray for $48 \mathrm{~h}$, according to the instruction of PI Cell Cycle Detection Kit, cell cycle was detected by flow cytometry (FCM) after cells were washed by PBS for one time, added with PI for 15 min of staining.

\section{Detection of the expression of apoptosis-related gene}

Western blotting was adopted to detection the protein levels of apoptosis-promoting genes (Bax and Cleaved caspase-3) and apoptosis-inhibiting gene (Bal-2). Cells from control group, Ad-null group and Ad-SIRT6 after irradiated by 4 Gy X-ray for $48 \mathrm{~h}$ were performed with protein extraction and SDS-PAGE. The operational approach was the same as the abovementioned Transfection efficiency test. Dilution rate of Bax and Caspase-3 was 1: 200 and dilution rate was 1: 300. And the final result was expressed as the ratio of SIRT6 to internal reference GAPDH band optical density.

\section{Statistical data analysis}

Windows SPSS16.0 software was used for data analysis. Quantitative data was expressed by the mean \pm standard deviation $(\overline{\mathrm{x}} \pm \mathrm{s})$. One-way Anova was used for comparison of multiple groups and SNK method was used for pair-wise comparison. A value of $P<0.05$ was considered to be significant.

\section{Results}

Effect of Ad-SIRT6 transfection on the expression of target gene

In the range of 25 400 pfu/cell, SIRT6 level of A549 
Table 1. SF of 3 Groups in Different Irradiation Doses (\%)

\begin{tabular}{lrccccc}
\hline \multirow{2}{*}{ roups } & \multicolumn{6}{c}{ Irradiation dose $(\mathrm{Gy})$} \\
\cline { 2 - 7 } & \multicolumn{1}{c}{0} & \multicolumn{1}{c}{4} & \multicolumn{6}{c}{6} & 8 & 10 \\
\hline Control group & $100.00 \pm 1.32$ & $93.16 \pm 1.74$ & $72.33 \pm 2.25$ & $55.37 \pm 1.79$ & $39.82 \pm 3.11$ & $14.28 \pm 1.46$ \\
Ad-null group & $99.01 \pm 2.16$ & $94.82 \pm 2.45$ & $70.48 \pm 1.97$ & $56.42 \pm 2.23$ & $37.69 \pm 2.57$ & $16.33 \pm 2.15$ \\
Ad-SIRT6group & $97.82 \pm 2.27$ & $78.65 \pm 1.86$ & $45.70 \pm 2.53^{\mathrm{ab}}$ & $24.59 \pm 1.82^{\mathrm{ab}}$ & $11.24 \pm 1.69^{\mathrm{ab}}$ & $2.75 \pm 1.02^{\mathrm{ab}}$ \\
\hline
\end{tabular}

Compared with control group, ${ }^{a} P<0.05$; Compared with Ad-null group, ${ }^{b} P<0.05$

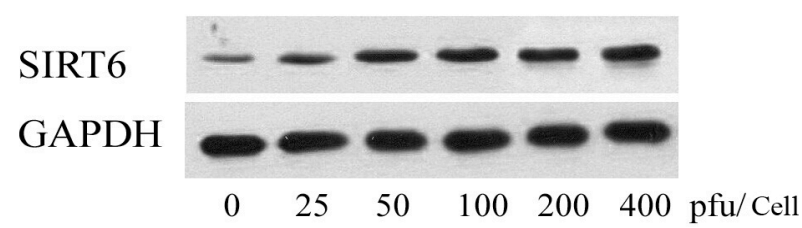

Figure 1. Effect of Ad-SIRT6 Transfection on Expression of the Target Gene

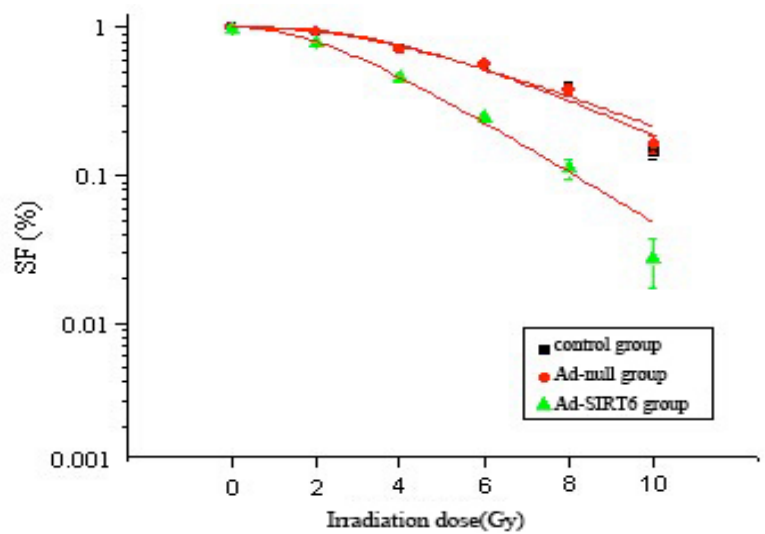

Figure 2. Multi-Target Single-Hit Model Fitting Survival Curve
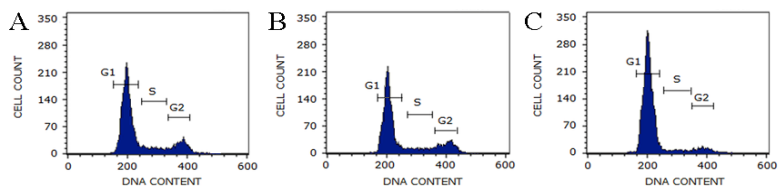

Figure 3. FCM Detection Figure of Cell Cycle of $\mathbf{3}$ Groups. A: control group; B: Ad-null group; C: Ad-SIRT6 group

cells increased as adenovirus concentration raised. The relative expression levels under the concentrations of $0,25,50,100,200$, and $400 \mathrm{pfu} /$ cell were $(0.12 \pm 0.02)$, $(0.15 \pm 0.03),(0.21 \pm 0.04),(0.38 \pm 0.04),(0.54 \pm 0.02)$ and $(0.67 \pm 0.04)$ and there were statistical differences among groups $(P<0.05)$ (Figure 1).

\section{Inhibiting effect of cell proliferation}

In the range of $25 \sim 400 \mathrm{pfu} / \mathrm{cell}$, the inhibitory rate of A549 cell proliferation increased as adenovirus concentration raised. The inhibitory rates under the concentrations of $0,25,50,100,200$, and $400 \mathrm{pfu} / \mathrm{cell}$ were $0 \%,(4.23 \pm 0.34) \%,(12.74 \pm 2.57) \%,(22.60 \pm 3.38) \%$, $(32.18 \pm 3.22) \%,(38.66 \pm 4.09) \%$ and $(47.81 \pm 5.58) \%$ and there were significantly differences among groups $(P<0.05)$.

\section{Radiosensitivity}

There was no difference between Ad-null group and control group after via the doses of 0,2, 4, 6, 8 and 10Gy $\mathrm{X}$-ray irradiation $(P>0.05)$. SF in Ad-SIRT group was
Table 2. Cell Distribution of Different Cell Cycle of 2 Groups after $\mathbf{4 8}$ h of $\mathbf{4 G y}$ X-ray Irradiation (\%)

\begin{tabular}{lllc}
\hline Groups & \multicolumn{1}{c}{$\mathrm{G} 1$} & \multicolumn{1}{c}{$\mathrm{S}$} & $\mathrm{G} 2$ \\
\hline Control group & $56.02 \pm 2.13$ & $27.16 \pm 1.35$ & $16.33 \pm 2.02$ \\
Ad-nullgroup & $54.24 \pm 1.86$ & $29.84 \pm 2.10$ & $17.48 \pm 1.97$ \\
Ad-SIRT6 group & $80.37 \pm 4.46^{\mathrm{ab}}$ & $14.58 \pm 1.14^{\mathrm{ab}}$ & $6.62 \pm 0.94^{\mathrm{ab}}$ \\
\hline
\end{tabular}

Compared with control group, ${ }^{a} P<0.05$; compared with Adnull group, ${ }^{b} P<0.05$

Table 3. Effect of Ad-SIRT6 on the Expression Level of Apoptosis-Related Genes

\begin{tabular}{lccc}
\hline Groups & \multicolumn{1}{c}{ Bcl-2 } & \multicolumn{1}{c}{ Bax } & Cleaved caspase-3 \\
\hline Control group & $0.52 \pm 0.04$ & $0.33 \pm 0.05$ & $0.36 \pm 0.04$ \\
Ad-null group & $0.38 \pm 0.05$ & $0.47 \pm 0.04$ & $0.52 \pm 0.06$ \\
Ad-SIRT6 group & $0.24 \pm 0.06^{\mathrm{ab}}$ & $0.61 \pm 0.04^{\mathrm{ab}}$ & $0.65 \pm 0.03^{\mathrm{ab}}$ \\
\hline
\end{tabular}

Compared with control group, ${ }^{a} P<0.05$; compared with Adnull group, ${ }^{b} P<0.05$

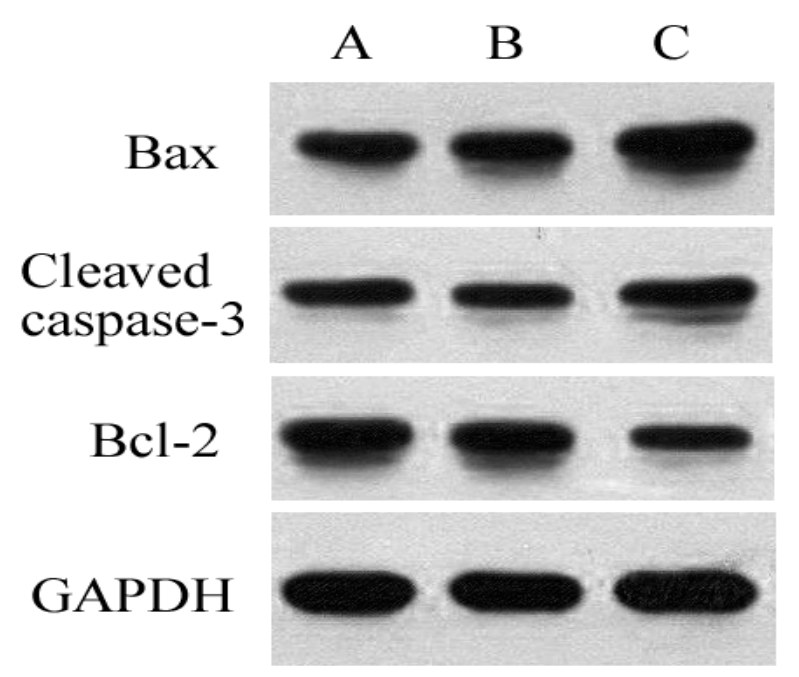

Figure 4. Western Blotting Kymogram of ApoptosisRelated Genes of 3 Groups. A: control group; B: Ad-null group; C: Ad-SIRT6 group

lower than Ad-null and control groups after 4 10Gy X-ray irradiation $(P<0.05)$. Multi-target single-hit model fitting survival curve was used for calculating SER (Figure 2). Control group D0 was (3.35 \pm 0.48$) \mathrm{Gy}$ and Ad-null group D0 was $(2.49 \pm 0.17) \mathrm{Gy}$, so the SER was 1.35 . (Table 1, Figure 2).

\section{Effect of Ad-SIRT6 transfection on cell cycle}

There was no difference in cell distribution of different cell cycle between control group and Ad-null group $(P>0.05)$. Compared with control and Ad-null groups, there was relatively higher cell proportion in phase G1 and lower cell proportion in phase $\mathrm{S}$ and $\mathrm{G} 2$ in Ad-SIRT6 group after $48 \mathrm{~h}$ of $4 \mathrm{~Gy} \mathrm{X}$-ray irradiation $(P<0.05)$ (Table 2 and Figure 3 ). 


\section{Effect of Ad-SIRT6 on the cell apoptosis}

There was no difference in expression level of apoptosis-related genes between control group and Adnull group $(P>0.05)$. Compared with other 2 groups, there were decreased level of Bal-2 and increased levels of Bax, Cleaved caspase-3 in Ad-SIRT6 group after 48 h of 4Gy $\mathrm{X}$-ray irrradiation $(P<0.05)$ (Table 3 and Figure 4$)$.

\section{Discussion}

NSCLC patients take up 80\% 85\% of total cases in lung cancer (Mutlu et al., 2013; Oven et al., 2013; Aydiner et al., 2013; Cai et al., 2013; Kaya et al., 2013; Natukula et al.,2013; Unal et al., 2013; Wang et al., 2013) and patients with locally advanced NSCLC who can't receive surgery were mainly treated with radiotherapy, the effect of which was mainly marked with dose-dependence. Although highdose radiotherapy can improve the remission rate and local control rate, at the same time, it can bring about severe side effects, such as severe radiation esophagitis and radiation pneumonitis (Feng et al.,2011). Radioresistance in tumor tissues limits its clinical application and hinder the improvement of cure rate of malignant tumors $(\mathrm{Ni}$ et al., 2013). Anoxia in solid tumor is the main cause of radiotherapy failure, so overcoming the radioresistance caused by anoxia is the key path for improving the therapeutic effect of the tumor (Wu et al., 2013). In order to adapt the circumstance of anoxia, glycometabolism of cancer cell can transform into glycolysis and production rate of glucose catabolism and lactic acid are improved by upregulating the level and activity of glycolytic enzyme, thus providing the material basis for the growth and malignant development of the tumor (Fantin et al., 2006; Bonnet et al., 2007; Le et al., 2010).

Sirtutin is a kind of NAD+-dependent histone deacetylase which is capable of changing target protein activity by lysine acetylation (Zhong et al., 2010; Sebastián et al., 2012). Mammal genome encode 7 broad-expression sirtutin subtypes (SIRT1 SIRT7), SIRT1 SIRT7 which play important roles in multiple physiological processes such as the growth and apoptosis of cells, of which, SIRT6 is a chromosome condensation factor for maintaining genomic stability (Sundaresan et al., 2012). SIRT6, located in telomere of human cells, can control cell aging when combined with the ninth lysine in acetylated histone H3 (Finkel et al., 2009). SIRT6 also plays an important role in energy regulation and can be considered as tumor suppressor gene, and once the defect of SIRT6 occurs, cells have oncogenicity (Sebastián et al., 2012). In this study, the overexpression of SIRT6 by adenovirus vector transtection of A549 cells was study and MTT assay showed that in the range of 25 400 pfu/ cell, the inhibitory rate of A549 cell proliferation increased as adenovirus concentration raised, revealing inhibiting effect of overexpression of SIRT6 on the growth of A549 cells.

This study employed multi-target single-hit model fitting drawing cell survival curve for calculating SER to evaluate the radiosensitization effect and the result showed SF in Ad-SIRT group reduced after A549 cells were irradiated by 4 10Gy X-ray, suggesting over-expressed
SIRT6 could improve the irradiation effect of X-ray. Moreover, when compared with control group, SER of AdSIRT6 was 1.35, which further proved that over-expressed SIRT6 had the radiosensitization effect. Cells in different phase of cell cycle have different sensitivity to radioactive rays. Phase $S$ is the least sensitive, followed by phase G1 and phase G2 and M are the most sensitive (Huang et al., 2008). In this study, compared with control and Ad-null groups, there was relatively higher cell proportion in phase G1 and lower cell proportion in phase S and G2 in AdSIRT6 group after $48 \mathrm{~h}$ of $4 \mathrm{~Gy} \mathrm{X}$-ray irradiation $(P<0.05)$, showing that over-expressed SIRT can inhibiting the process of cell cycle manifested with retarded phase G1, delayed phase $\mathrm{G} 2$ and $\mathrm{S}$, prolonged cell doubling time and slow rate of cell proliferation, consequently enhancing overall radiosensitivity of the tumor.

In addition, the effect of overexpression of SIRT6 on the cell apoptosis was studies in this paper, in which Bax and Cleaved capspase- 3 are apoptosis-promoting gene and Bal-2 is common apoptosis-inhibiting gene (Liu et al., 2010; Kim et al., 2012). The levels of these three kinds of proteins are commonly used to evaluate the status of cell apoptosis. The result in this study compared with control group and Ad-null group, there were decreased level of Bal-2 and increased levels of Bax and Cleaved caspase-3 in Ad-SIRT6 group after 48 h of 4Gy X-ray irrradiation $(P<0.05)$, revealing over-expressed SIRT6 could promote the apoptosis of A549 cells.

In conclusion, in NSCLC cell lines cultured in vitro, over-expressed SIRT6 by adenovirus vector, which has radiosensitization effect on A549 cells, can reduce the ability of cell proliferation, change the cell distribution of cell cycle and induce the cell apoptosis, for those reasons, can be as an adjuvant therapy in the treatment of locally advanced NSCLC, with application prospects.

\section{References}

Aydiner A, Ciftci R, Karabulut S, et al (2013). Does beta-blocker therapy improve the survival of patients with metastatic non-small cell lung cancer? Asian Pac J Cancer Prev, 14, 6109-14.

Bonnet S, Archer SL, Allalunis-Turner J, et al (2007). A mitochondria- $\mathrm{K}+$ channel axis is suppressed in cancer and its normalization promotes apoptosis and inhibits cancer growth. Cancer Cell, 11, 37-51.

Cai Y, Wang JY, Liu H (2013). Clinical observation of whole brain radiotherapy concomitant with targeted therapy for brain metastasis in non-small cell lung cancer patients with chemotherapy failure. Asian Pac J Cancer Prev, 14, 5699-703.

Fantin VR, St-Pierre J, Leder P (2006). Attenuation of LDH-A expression uncovers a link between glycolysis, mitochondrial physiology, and tumor maintenance. Cancer Cell, 9, 425-34.

Finkel T, Deng CX, Mostoslavsky R (2009). Recent progress in the biology and physiology of sirtuins. Nature, 460, 587-91.

Feng R, Guo JS, He JX, et al (2011). CMNa affect radiosentivivity and homogenegous adhesion of Tca-8113 cell line. China Oncol, 21, 17-21.

Huang L, Wang RZ (2008). Cyclin and tumor radiosensitivity. J Xinjiang Med Univ, 31, 1510-2.

Jemal A, Siegel R, Xu J, et al (2010). Cancer statistics. $C A$ Cancer J Clin, 60, 277-300. 
Kaya V, Yildirim M, Demirpence O, et al (2013). Prognostic significance of basic laboratory methods in non- small-celllung cancer. Asian Pac J Cancer Prev, 14, 5473-6.

Kim SH, Jang YW, Hwang P, et al (2011). The reno-protective effect of a phosphoinositide 3-kinase inhibitor wortmannin on streptozotocin-induced proteinuric renal disease rats. Exp Mol Med, 44, 45-51.

Le A, Cooper CR, Gouw AM, et al (2010). Inhibition of lactate dehydrogenase A induces oxidative stress and inhibits tumor progression. Proc Natl Acad Sci USA, 107, 2037-42.

Liu ZG, Yu ZC, Yu YP, et al (2010). Lysophosphatidic acid level and the incidence of silent brain infarction in patients with nonvalvular atrial fibrillation. Int J Mol Sci, 11, 3988-98.

Ni XY, Qian N, Lin T, et al (2013). Radiosensitization effect of gold nanoparticles modified by sodium glycidazole on lung adenocarcioma cell A549. Chin J Radiol Med Protec, 33, 265-8.

Natukula K, Jamil K, Pingali UR, et al (2013). The codon 399 Arg/Gln XRCC1 polymorphism is associated with lung cancer in Indians. Asian Pac J Cancer Prev, 14, 5275-9.

Oven Ustaalioglu BB, Unal OU, Turan N, et al (2013). Prognostic factors for lymph node negative stage I and IIA non-small cell lung cancer: multicenter experiences. Asian Pac J Cancer Prev, 14, 6287-92.

Sundaresan NR, Vasudevan P, Zhong L, et al (2012). The sirtuin SIRT6 blocks IGF-Akt signaling and development of cardiac hypertrophy by targeting c-Jun. Nat Med, 18, 1643-50.

Sebastián C, Zwaans BM, Silberman DM, et al (2012). The histone deacetylase SIRT6 is a tumor suppressor that controls cancer metabolism. Cell, 151, 1185-99.

Unal D, Eroglu C, Kurtul N, et al (2013). Are neutrophil/ lymphocyte and platelet/lymphocyte rates in patients with non-small cell lung cancer associated with treatment response and prognosis? Asian Pac J Cancer Prev, 14, 5237-42.

Vokes EE, Herndon JE II, J, Kelley MJ, et al (2007). Induction chemotherapy compared with chemoradiotherapy alone for regionally advanced unresectable stage III non-small-cell lung cancer: Cancer and Leukemia Group B. J Clin Oncol, 25, 1698-704.

Vander Heiden MG, Cantley LC, Thompson CB (2009). Understanding the Warburg effect: the metabolic requirements of cell proliferation. Science, 324, 1029-33.

Wang JY, Cai Y (2013). Clinical observation and prognostic analysis of pemetrexed plus platinum as first-line treatment in patients with advanced non-small cell lung cancer. Asian Pac J Cancer Prev, 14, 6267-71.

Wu GY, Hou PC, Wang W (2013). Radioresistance change and the machanism of human esophaged cancer EC706 cells in hypoxia. Chin J Radiol Med Protec, 33, 138-41.

Zhong L, D'Urso A, Toiber D, et al (2010). The histone deacetylase Sirt6 regulates glucose homeostasis via Hif1alpha. Cell, 140, 280-93. 